\title{
Prevenção de doenças sexualmente transmissíveis na visão de idosos de uma Estratégia da Saúde da Família
}

\author{
Prevention of sexually transmitted diseases in the point of view of elderly clients of a Family Health Strategy \\ Prevención de enfermedades sexualmente transmisibles según la óptica de ancianos \\ de una Estrategia de Salud Familiar
}

\section{Andreia Kullmann Cezar', Marinês Aires", Adriana Aparecida Paz"I}

\author{
' Faculdade Nossa Senhora de Fátima, Curso de Especialização de Enfermagem em Terapia Intensiva. \\ Caxias do Sul-RS, Brasil.
}

"Universidade Regional e Integrada do Alto Uruguai, Campus Frederico Westphalen. Frederico Westphalen-RS, Brasil.

"' Universidade Federal do Rio Grande do Sul, Programa de Pós Graduação em Enfermagem (Doutoranda). Porto Alegre-RS, Brasil.

\author{
Submissão: 13-02-2011 Aprovação: 13-11-2012
}

\section{RESUMO}

O objetivo deste trabalho foi o de avaliar o conhecimento de pessoas idosas sobre as ações preventivas para as doenças sexualmente transmissíveis (DSTs) no contexto da Estratégia Saúde da Família (ESF). Trata-se de um estudo transversal, envolvendo 94 pessoas idosas, idade $\geq 60$ anos, adstritas à ESF na Serra Gaúcha. Os resultados apontam paridade na amostra para vida sexual ativa e predominância da atividade sexual com o mesmo parceiro. As pessoas idosas têm conhecimento de como evitar as DSTs, sendo enfático o uso de preservativos. A maioria relatou que não recebeu orientações da equipe da ESF; já os idosos que receberam, os mesmos declararam que a orientação teve o enfoque no preservativo. É necessário intensificar as ações e discussões em torno da sexualidade e DSTs, visando ao envelhecimento saudável.

Descritores: Programa Saúde da Família; Saúde do Idoso; Doenças Sexualmente Transmissíveis; Educação em Saúde.

\section{ABSTRACT}

The aim of this study was to assess the knowledge of elderly people on preventive actions to sexually transmitted diseases (STDs) in the context of the Family Health Strategy (FHS). This is a cross-sectional study, involving 94 elderly, aged $\geq 60$ years, attached to the ESF in Serra Gaucha (a region at Rio Grande do Sul, Brazil). The results indicate parity in the sample for sexual activity and prevalence of sexual activity with the same partner. Older people have knowledge of how to prevent STDs, mostly by the use of condoms. Most interviewees reported they received no information from FHS team. Those who received orientation related that the focus was on the usage of condoms. It is necessary to intensify the actions and discussions of sexuality and STDs, aimed at healthy aging.

Key words: Family Health Program; Health of the Elderly; Sexually Transmitted Diseases; Health Education.

\section{RESUMEN}

El objetivo de este trabajo fue la evaluación de conocimiento de personas mayores con respecto a acciones de prevención para enfermedades sexualmente transmisibles en el contexto de la Estrategia Salud de la familia (ESF). Se trata de un estudio transversal con 94 personas con más de 60 años asociadas a ESF, en la Sierra Gaucha (Rio Grande do Sul, Brasil). Los resultados demuestran paridad en relación a la vida sexual activa y predominancia de la vida sexual con la misma pareja. Los ancianos tienen conocimiento sobre las formas de evitar las enfermedades sexualmente transmisibles y son enfáticos sobre el uso de preservativos. La mayoría relató que no recibió orientación del equipo de ESF; los que recibieron dijeron que las orientaciones tuvieron enfoque en el uso del preservativo. Es necesario intensificar las acciones y discusiones sobre la sexualidad y enfermedades sexualmente transmisibles pensando en la vejez saludable.

Palabras clave: Programa de Salud Familiar; Salud del Ancian; Enfermedades de Transmisión Sexual; Educación en Salud.

\section{AUTOR CORRESPONDENTE Adriana Aparecida Paz E-mail: adrianap@ufcspa.edu.br}




\section{INTRODUÇÃO}

No Brasil, a implantação do Sistema Único de Saúde (SUS) pressupõe a adoção de um modelo assistencial de Vigilância à Saúde que atenda às diretrizes e aos princípios expressos na Constituição Federativa Brasileira de 1988. Posteriormente, a Estratégia de Saúde da Família (ESF) surgiu para reorganizar os serviços de saúde, configurando a implementação da Política de Atenção Básica, em 2002. Tal política é definida como um conjunto de ações de saúde, de caráter individual e coletivo, pautada por estratégias de promoção, proteção da saúde, prevenção de agravos, diagnóstico precoce, tratamento, limitação da incapacidade, reabilitação e manutenção da saúde ${ }^{(1)}$.

Historicamente, a Declaração de Alma Ata, em 1978, estimulou os países a elencar as ações de atenção primária, de modo a propiciar a adesão dos indivíduos a estilos de vida saudáveis em todas as fases do ciclo vital. A saúde se impõe como direito fundamental de todo cidadão, se constituindo na principal meta de cunho social de todos os governos. Portanto, torna-se indispensável que a população adquira uma consciência crítica quanto ao envelhecimento saudável, para que assegure práticas de proteção à saúde, tendo em vista que suas próprias ações e atitudes que pode deflagrar em adoecimento $^{(2)}$.Tal situação de adoecimento carece de ações para controle e redução de danos à saúde que demandam por recursos do sistema de saúde.

A promoção da saúde do idoso aponta para a necessidade de um processo de capacitação de indivíduos numa perspectiva coletiva, visando à melhoria das condições de vida e de saúde ${ }^{(3)}$. Por sua vez, essas ações resultam da combinação de estratégias elencadas pelos gestores com respaldo das políticas públicas. A Política Nacional de Saúde da Pessoa Idosa (PNSPI) foi instituída pela Portaria ${ }^{\circ} 2.528 / 06$, com a finalidade de recuperar, manter e promover a autonomia e a independência da pessoa idosa por meio de medidas individuais e coletivas, em consonância com os princípios e diretrizes do SUS. Nessa perspectiva, o conceito de saúde para a pessoa idosa é a plena condição de autonomia e independência para a tomada de decisões, quer pela presença ou ausência de morbidades ${ }^{(4)}$. Dentre as diretrizes da PNSPI e as prioridades do Pacto pela Vida, destaca-se a promoção do envelhecimento ativo e saudável. Nessa acepção, a promoção da saúde apresenta-se como a interface das ações de educação com as de saúde, traduzindo-se em ações de cunho de preventivo. Para tanto, as práticas de educação em saúde no contexto da ESF carecem de propostas que busquem diferenciar-se do modelo tradicional de educação em saúde, o qual visa à prevenção de doenças de maneira isolada, o que não privilegia a preponderância das ações de cunho coletivo decorrente da inter-relação do homem e o ambiente.

Nesse contexto, agregam-se ainda as mudanças demográficas e epidemiológicas no cenário brasileiro que são observadas pelo aumento populacional de pessoas idosas e de agravos de longa duração. Esses eventos são comprovados por numerosos estudos $^{(5-7)}$ que mencionam os desafios que são e poderão ser enfrentados pela família, pelos serviços de saúde e pela sociedade. No Brasil, as estimativas populacionais apontaram que em 2009, 10,1\% da população é composta por pessoas com idade $\geq 60$ anos; já no Rio Grande do Sul (RS), esse percentual eleva-se para $12,9 \%{ }^{(8)}$. Os dados epidemiológicos constatam o aumento progressivo no número de casos de doenças sexualmente transmissíveis (DSTs), em especial pelo vírus da imunodeficiência humana (HIV), entre as pessoas com idade de 50 a 70 anos $^{(9)}$.

Face ao exposto, vislumbra-se uma lacuna no pensar e agir dos profissionais de saúde quando o assunto é a sexualidade das pessoas idosas. Tal atividade foi historicamente negada ou anulada por valores e normas socioculturais com enfoque em estereótipos negativos vinculados ao envelhecimento humano. Entretanto, com o aumento da expectativa de vida e da disponibilidade de novas tecnologias, torna-se possível prolongar a vida, ao mesmo tempo em que se almeja também ampliar a qualidade de vida aos idosos. A sexualidade emerge no cenário da atenção à saúde do idoso como um complexo problema de Saúde Pública, pois esta se torna sobressalente não pela sua negligência ou anulação sócio-histórica, mas pelas DSTs que são adquiridas pelo convívio sexual com outros parceiros de mesma faixa etária ou ainda mais jovens ${ }^{(10)}$.

Diversos fatores contribuem para esse aumento de DSTs entre as pessoas idosas. Tal fato vem se destacando em função de a sociedade ignorar que os idosos mantêm a vida sexual ativa; da escassez de estratégias e orientações que priorizem a prevenção de DSTs na atenção básica; e das dificuldades motoras dos idosos no uso de preservativos. Desta maneira, esses fatores supracitados denotam as fragilidades das estratégias de saúde diante da magnitude e da vulnerabilidade da pessoa idosa para as DSTs ${ }^{(11)}$. Por outro lado, é preciso considerar que outros fatores permitem observar que os princípios e as diretrizes do SUS estão sendo empregados, observando-se a potencialidade dos serviços de saúde em decorrência do maior empenho dos profissionais na notificação de casos suspeitos; e que os recursos farmacológicos e tecnológicos possibilitam prolongar a vida na medida em que ocorre o envelhecimento de pessoas portadoras de DSTs.

Nessa perspectiva, emergem novas questões para o campo da prática de saúde em relação às abordagens da sexualidade, do acesso aos serviços e das ações preventivas estabelecidas no contexto da ESF. Na literatura, esta questão da sexualidade biológica na fase idosa ainda é pouco explorada no que se refere à prevenção das DSTs. Deste modo, surgiu essa investigação que pretende contribuir com outros estudos, de tal forma que se promovida a investigação, a reflexão e ações que consolidem o conhecimento e novas propostas capazes de suscitar discussões na esfera da atenção básica. O objetivo do estudo foi o de avaliar o conhecimento de pessoas idosas sobre as ações preventivas para as doenças sexualmente transmissíveis (DSTs) no contexto da Estratégia Saúde da Família (ESF).

\section{MÉTODOS}

A pesquisa caracterizou-se como transversal, contemplando uma abordagem quantitativa. O cenário de estudo foi uma unidade de ESF, em um município da região da Serra Gaúcha. A estimativa de habitantes para o município, no ano de 2009, 
era de 411.167 habitantes, dentre os quais $10,3 \%$ da população pertenciam à faixa etária $\geq 60 \operatorname{anos}^{(8)}$. A unidade tem três equipes de ESF e duas equipes de apoio em odontologia. $\mathrm{O}$ estudo foi realizado nesta unidade por apresentar um maior número de idosos (em torno de $12 \%$ ) a ela vinculados.

A população em estudo foi constituída por pessoas idosas com idade $\geq 60$ anos. Os critérios de inclusão foram: idade $\geq 60$ anos e residir em área de abrangência da ESF. Durante o período de coleta de dados, foram abordadas 146 pessoas idosas que procuraram atendimento na unidade, apenas 94 $(64,3 \%)$ aceitaram participar do estudo, sendo constituída a amostra por conveniência, o que representou $16,2 \%$ do total de pessoas idosas adstritas à área de abrangência da unidade. Os motivos elencados da não participação no estudo foram expressos por $52(35,6 \%)$ pessoas idosas, que justificaram a falta de disponibilidade para a entrevista por conta do horário de trabalho ou de compromissos com atividades domésticas. Desse modo, foi oportunizado um agendamento para que os idosos retornassem à unidade para participar do estudo, mas não houve o retorno no período da coleta de dados.

Atendendo aos critérios de elegibilidade do estudo, a pessoa idosa foi encaminhada a uma sala com privacidade, sem fluxo de profissionais, para realizar a leitura e assinatura do termo de consentimento livre e esclarecido, em duas vias, no qual constaram as informações sobre os objetivos, a justificativa, os procedimentos, os riscos e os benefícios do estudo. A partir disto, os dados foram coletados por meio de uma entrevista estruturada com a utilização de um instrumento, sendo contempladas as dimensões demográficas, socioeconômicas, situação de saúde e sexualidade das pessoas idosas. Destaca-se que, para utilizar o instrumento elaborado pelas pesquisadoras, foi realizado um pré-teste com cinco pessoas idosas que visava avaliar a compreensão e clareza das questões, para que houvesse a adequação das mesmas.

Posteriormente, os dados coletados foram organizados e codificados nos instrumentos e a seguir, os mesmos foram registrados por meio da digitação no banco de dados do programa SPSS 16.0, o qual foi utilizado para o gerenciamento da análise dos dados. As variáveis foram analisadas pela estatística descritiva, em que as variáveis contínuas são apresentadas por meio de média e desvio padrão; já as variáveis nominais são expressas por frequência absoluta e relativa ${ }^{(12)}$. $\mathrm{O}$ estudo foi aprovado pelo Comitê de Ética e Pesquisa da Associação Cultural e Científica Nossa Senhora de Fátima (CEP-FÁTIMA), sob o número 025/08 de acordo com as Diretrizes e Normas Regulamentadoras de Pesquisa envolvendo Seres Humanos, do Conselho Nacional de Saúde ${ }^{(13)}$.

\section{RESULTADOS E DISCUSSÃO}

\section{Caracterização da amostra estudada}

Na Tabela 1, são apresentados os dados das 94 pessoas idosas que participaram do estudo quanto às características demográficas e socioeconômicas. O estudo apresentou uma distribuição semelhante dos sexos das pessoas idosas que buscaram o atendimento na unidade. Na avaliação das estimativas populacionais para 2009, a prevalência é do sexo feminino em 57,6\% no cenário do estudo ${ }^{(8)}$. Estudo realizado no Rio de Janeiro ${ }^{(14)}$ observou que as mulheres procuram utilizar regularmente os serviços de saúde, diferentemente do que acontece entre os homens. Além disso, as mulheres procuram realizar os exames de rotina e prevenção, e os homens procuram atendimento mais por motivo de doença. Assim, as características do serviço podem provocar diferenças na busca de atendimentos para a população assistida. Em cidades com unidades de saúde específicas para DSTs, prevalece a demanda masculina. Em contrapartida, quando ocorre a atenção em unidades convencionais de saúde, a demanda maior é de mulheres ${ }^{(14)}$. Contudo, considerou-se como uma limitação do estudo o desconforto das mulheres para falar sobre a sexualidade e DSTs. Tal temática abordada pode ter sido decisiva para as recusas na participação neste estudo, ocorrendo, assim, uma maior participação masculina.

Tabela 1 - Características demográficas e socioeconômicas de pessoas idosas adstritas a Estratégia da Saúde da Família, em um município da Serra Gaúcha, 2009.

\begin{tabular}{lc}
\hline Variáveis demográficas e socioeconômicas & $\mathbf{N}(\%)$ \\
\hline Sexo (masculino) & $48(51,1)$ \\
Idade (anos) & $67,6(6,6)^{*}$ \\
Situação conjugal & \\
$\quad$ Casado (a) ou vive com companheiro(a) & $50(53,2)$ \\
$\quad$ Solteiro(a) ou viúvo(a) & $32(34,0)$ \\
$\quad$ Divorciado(a), separado(a), desquitado(a) & $12(12,8)$ \\
Filhos (sim) & $91(96,8)$ \\
Escolaridade (sim) & $82(87,2)$ \\
Anos de estudo & \\
$\quad 1$ ト 5 anos & $48(58,5)$ \\
5 F 9 anos & $27(32,9)$ \\
$\quad \geq 9$ anos & $7(8,6)$ \\
Participação em atividade social & $60(63,8)$ \\
Beneficiário do INSS & $70(74,5)$ \\
\hline
\end{tabular}

*Dado apresentado em média \pm desvio padrão Fonte: Dados da pesquisa.

Houve a predominância da faixa etária dos 60 aos 69 anos, o que corroborou as estimativas populacionais, para o período de 2009, no município cenário do estudo, sendo estimado que $57,3 \%$ dos idosos estão nessa faixa etária dos 60 aos 69 anos $^{(8)}$. O crescimento da população idosa, no Brasil, vem ocorrendo de forma bastante acelerada, pois a faixa etária dos 60 aos 69 anos é a que mais cresce na maioria dos países em desenvolvimento $^{(15)}$. A situação conjugal para a maioria dos idosos é a casada, seguidos de viúvos, e com predomínio de 96,8\% de idosos que tiveram filhos. Em relação ao tipo de situação conjugal dos idosos, esta pode se dar em decorrência do aumento progressivo na expectativa de vida da população e pelos fatores morais de que o casamento é somente um para a vida inteira ${ }^{(16)}$. As mesmas autoras ${ }^{(16)}$ observaram entre os idosos assistidos por 
uma ESF que $45,3 \%$ eram casados e $44 \%$ viúvos.

No que se refere à escolaridade, $87,2 \%$ dos idosos são alfabetizados e, dentre estes, 58,5\% estudaram no máximo quatro anos. A situação da escolaridade das pessoas idosas hoje se remete ao período da década de 20 a 50, quando a oportunidade de acesso à educação ocorria de forma assimétrica por classe social e gênero, tendo como consequência a dificuldade de se buscar condições de existência e sobrevivência, principalmente, por parte do gênero feminino ${ }^{(16)}$. Em relação à aposentadoria, $74,5 \%$ dos idosos declararam ter essa condição, e 77,8\% possuem uma renda familiar de até três salários mínimos. A situação empregatícia ocorre para 31,5\% dos idosos empregados formalmente no mercado de trabalho; $13,7 \%$ possuíam atividade autônoma regular, 12,9\% estavam desempregados e 19,5\% eram aposentados ${ }^{(18)}$. A participação em atividades sociais foi declarada por $63,8 \%$ dos idosos, que destacaram participar das atividades da igreja; dos grupos de idosos; clube de mães; cursos de aperfeiçoamento; bailes da terceira idade; associação de moradores, entre outros. As atividades sociais podem proporcionar momentos de prazer como apontado em outro estudo $^{(17)}$, em que 56,4\% de pessoas idosas realizam atividades de recreação e lazer. Logo, as características demográficas e socioeconômicas identificadas e discutidas permitem inferir que, ao conhecer a realidade social das pessoas idosas no contexto da ESF, cria-se a possibilidade de se elencar estratégias e espaços para discussão, no sentido de se apoiar e reduzir os fatores que possam influenciar negativamente na qualidade de vida e envelhecimento ativo da população.

\section{Sexualidade e a Prevenção de DSTs no envelhecimento}

Os resultados relacionados à sexualidade e ao conhecimento para a prevenção de DSTs são apresentados na Tabela 2. Os dados apontam para a paridade na amostra quanto ao relato de vida sexual ativa e não ativa, sendo predominante a atividade sexual com o mesmo parceiro. Os achados deste estudo se contrapõem aos dados de outro estudo ${ }^{(19)}$ em que se observou que atividade sexual ativa foi relatada por $48,5 \%$ das mulheres e por 30,6\% dos homens idosos. No entanto, corroboram o mesmo estudo ${ }^{(19)}$ no que tange à atividade sexual com o mesmo parceiro, o que foi relatado por $72,8 \%$ dos idosos. Tem-se observado que o envelhecimento da população, e o aumento do número de pessoas idosas, acompanhado da melhoria da qualidade de vida, possibilita mudanças de comportamento relacionadas à sexualidade ${ }^{(20-2)}$. Além disso, revela-se a forma excludente da maneira que vem sendo abordada a sexualidade das pessoas idosas ${ }^{(21,23)}$, como também a exclusão em projetos e programas de educação e prevenção de DSTs, o que demonstra uma grande lacuna nas múltiplas referências dos fatos em que constrói a magnitude das morbidades transmissíveis.

A maioria dos idosos declarou que sabe como evitar as doenças transmitidas pelo sexo e relataram que, sem o uso de preservativo, podem-se transmitir muitas doenças, como o HIV, que não tem cura. As pessoas idosas foram questionadas sobre a idade para se evitar DSTs, sendo relatado que a prevenção deve ocorrer desde a primeira atividade sexual 48,9\%, seguida da fase em que o indivíduo está na adolescência 30,9\%. Em relação ao comportamento sexual, mostrou-se que a maior
Tabela 2 - Sexualidade e conhecimento para a prevenção de DSTs autorreferido pelas pessoas idosas adstritas à Estratégia da Saúde da Família, em um município da Serra Gaúcha, 2009.

\begin{tabular}{lr}
\hline Sexualidade e conhecimento para a prevenção de DSTs & \multicolumn{1}{c}{ n(\%) } \\
\hline Atividade sexual (sim) & $47(50,0)$ \\
Ocorre com a mesma pessoa (sim) & $45(95,7)$ \\
Sabe como evitar DSTs (sim) & $93(98,9)$ \\
Tem alguma DSTs (não) & $94(100,0)$ \\
Recebeu orientações da equipe de enfermagem da ESF $(\operatorname{sim})$ & $23(24,5)$
\end{tabular}

Fonte: Dados da pesquisa.

parte da população teve sua iniciação sexual entre 14 e 19 anos $(65,2 \%)$,e que os mesmos não usaram preservativo no passa$\mathrm{do}^{(19)}$. Em outro estudo ${ }^{(24)}, 86,3 \%$ dos idosos entrevistados, em uma amostra de 510, declararam que não usavam preservativos. No momento das entrevistas, todos os idosos declararam não serem portadores de DSTs. Esses achados contrapõem-se aos de outro estudo ${ }^{(23)}$ que constataram a magnitude para DSTs, a qual atinge pessoas com idade superior a 60 anos sendo este, portanto, um problema de saúde pública.

A maioria dos idosos relatou que não houve a oportunidade de discutir ou receber orientações sobre a sexualidade e a prevenção de DSTs da equipe do ESF. Já para os idosos que tiveram a oportunidade desse espaço com a equipe descreveram que a orientação teve o enfoque no uso de preservativo, que o homem e a mulher transmitem doenças por meio do sexo, e que não existe cura para muitas DSTs. Outra fonte de informação que predominou para esses idosos para saber como evitar as DSTs é a televisão (41,4\%). E o método mais seguro, na visão dos idosos, é o uso do preservativo masculino (90,1\%).

Quanto às orientações da equipe de enfermagem, apenas $24,5 \%$ dos idosos relataram ter recebido orientações sobre DSTs, denotando que a Enfermagem precisa atuar de maneira mais incisiva nesta seara da saúde do idoso, como medida para estabelecer o cuidado integral diante DSTs, seja em consultas de enfermagem, visitas domiciliares ou em grupos da terceira idade. Um estudo ${ }^{(12)}$ aponta a existência da dificuldade pelos profissionais de saúde em falar sobre a sexualidade do idoso por ter seus conhecimentos direcionados para outros grupos específicos, adolescentes e adultos, que excluem o idoso desse cenário de cuidado. Por sua vez, muitas pesquisas abordam a ação educativa do enfermeiro para DSTs na adolescência, carecendo estudos que tragam a sexualidade e DSTs na área da saúde do idoso para valorizar a atuação da enfermagem nesse cenário de cuidado, assim como mostrar melhores resultados e impactos das intervenções sobre a saúde das pessoas idosas.

Constatou-se, neste estudo, na dimensão sexualidade do idoso, que a maioria não possui informações detalhadas sobre DSTs, tendo como referência apenas o uso de preservativos. Estes dados revelam que, no cerne da ESF, os usuários carecem de atenção especial para abordagem da sexualidade no enveIhecimento, sob a luz das políticas públicas sociais e de saúde, com o intuito de evitar a propagação de DSTs entre os indivíduos idosos. Face ao exposto, os temas sobre sexualidade, 
conhecimento e riscos são tratados enfaticamente para grupos específicos da população, como adolescentes e adultos em idade reprodutiva ${ }^{(10,21)}$. Os assuntos sobre sexualidade para a população idosa têm menor ênfase para ações preventivas devido ao fato de que não existe mais a preocupação com anticoncepção, sendo apenas tratados em decorrência de aspectos relativos ao desempenho ou às disfunções sexuais.

Todavia, este estudo aponta para a necessidade de desenvolver as habilidades pessoais de idosos como meio de ganho de saúde pelo empoderamento, com vistas a garantir a promoção da saúde, caracterizando por redução de indicadores de morbidade e mortalidade por DSTs. Indubitavelmente, é essencial a educação em saúde nesta perspectiva para que a pessoa idosa tenha a tomada de decisão sobre as melhores práticas de saúde alcançando a coletividade, de modo que reduza os fatores para ocorrência do adoecimento. Nesta concepção, a educação em saúde poderá propiciar a autoestima do idoso de modo que reduza a alienação e incremente os conhecimentos ao ponto de abrir o campo de escolhas do indivíduo para decidir sobre suas atitudes e comportamento.

\section{CONSIDERAÇÕES FINAIS}

O presente trabalho é extremamente relevante, pois observou a lacuna existente quanto à consolidação das ações de enfermagem para promover à saúde das pessoas idosas sob sua sexualidade e DSTs no cenário de cuidado da ESF. Com base neste estudo, observou-se um grande número de idosos que relatou não haver recebido orientações da enfermagem sobre a sexualidade envolvendo a prevenção de DSTs. Nesse sentido, os profissionais de saúde precisam abordar essa temática nos grupos de idosos, acolhimento e consultas, assim como em outros espaços de atenção à saúde do idoso. O enfermeiro precisa considerar a sexualidade do idoso como presente até a finitude, para que possa atingir as metas de cuidado para essa população por meio da intervenções que visem à prevenção de DSTs.

Acredita-se que a Enfermagem careça de educação permanente para consolidar os conhecimentos sobre a sexualidade do idoso, já que essa população vem aumentando e atingindo idade mais avançada. Tal educação oportuniza a obtenção de subsídios para que se proponha um cuidado integral ao idoso, de modo que possa atender e esclarecer os idosos sobre as possíveis dúvidas relacionadas à sexualidade, assim como estabelecer pelos próprios idosos com base no conhecimento adquirido pela educação os cuidados quanto ao ato sexual e as DSTs. Quiçá, nos currículos de graduação e pós-graduação, seja necessário um olhar especial para a inclusão de disciplinas na área de saúde do idoso, ou ainda que o envelhecimento seja abordado de forma transversal nas disciplinas que compõem a formação acadêmica. Tal incremento nos currículos poderá tornar os profissionais mais qualificados, que atendam a demanda populacional crescente de idosos no cenário brasileiro, no sentido de um envelhecimento ativo e saudável.

Ainda, torna-se importante o desenvolvimento de programas de saúde pública específicos às pessoas idosas para que se tenha esclarecimento das principais dúvidas relacionadas à sexualidade e à prevenção de DSTs. A partir dessas estratégias educativas, realizadas pela equipe de saúde, possibilita a adoção de práticas saudáveis pelas pessoas idosas para o ato sexual e prevenção das DSTs. Portanto, evidencia-se a necessidade de executar ações educativas direcionadas aos idosos em decorrência dos achados, dando início dessas ações na rede de atenção do município cenário deste estudo. Deste modo, cabe aos enfermeiros da atenção primária à saúde propor ações e aproveitar as oportunidades de contato com os idosos para trazer à discussão cuidados com a sexualidade, quanto aos fatores de risco e de proteção. Esses espaços de cuidado e de inserção do enfermeiro podem estabelecer intervenção diante da magnitude das DSTs por meio da consulta de enfermagem, visita domiciliar, acolhimento, sala de espera e/ou grupos.

A investigação realizada no serviço de saúde sobre a sexualidade e a prevenção para DSTs possibilitou produzir conhecimentos sobre a temática, e espera-se que esse estudo possa contribuir para o direcionamento de políticas de saúde, em especial, à pessoa idosa, para que seja possível planejar uma intervenção com ações futuras relacionadas às DSTs. Além disso, incontestavelmente, é necessário o incremento de pesquisas de cunho epidemiológico para se avaliar o conhecimento e comportamento sexual da população idosa sobre a prevenção das DSTs.

Face ao exposto neste estudo, ficou evidenciada a necessidade de ampliação das ações de enfermagem na atenção primária à saúde abordando a sexualidade das pessoas idosas em qualquer espaço de cuidado como possibilidade de reduzir os indicadores de morbidade e mortalidade de idosos por agravantes de DSTs.

\section{AGRADECIMENTO}

Agradecemos à Secretaria Municipal de Saúde de Caxias do Sul, Rio Grande do Sul, pela autorização e incentivo na realização deste estudo junto às pessoas idosas adstritas de uma Unidade de Estratégia de Saúde da Família.

\section{REFERÊNCIAS}

1. Ministério da Saúde, Secretaria de Atenção a Saúde, Departamento de atenção Básica. Política Nacional de Atenção Básica. 4. ed. Brasília: Ministério da Saúde; 2007.

2. Oliveira SMJV, Santos JLF, Lebrão ML, Duarte YAO, Pierin
AMG. Hipertensão arterial referida em mulheres idosas: prevalência e fatores associados. Texto Contexto \& Enferm 2008;17(2):241-9.

3. Ministério da Saúde. Anuário estatístico de saúde do Brasil 
2001 [monografia na internet]. Brasília: Ministério da Saúde; 2002. [acessado 08 out 2008]. Disponível em: < http:// portal.saude.gov.br/saude/aplicacoes/anuario2001/index. cfm >

4. Ministério da Saúde, Departamento de Informática do Sistema Único de Saúde. Sistema de Informação Hospitalar. [monografia na internet]. Brasília: Ministério da Saúde; 2006. [acessado em 10 maio 2009]. Disponível em: < http://tabnet.datasus.gov.br/cgi/deftohtm.exe?sih/cnv/nirs.def $>$

5. Silvestre JA, Costa Neto MM. Abordagem do idoso em programas de saúde da família. Cad Saúde Pública 2003;19(3):839-47.

6. Paz AA. Características de pessoas idosas em condição de alta hospitalar associadas à rehospitalização. Porto Alegre. Dissertação [Mestrado em Enfermagem]- Escola de Enfermagem, Universidade Federal do Rio Grande do Sul; 2004.

7. Aires M, Paz AA. Necessidades de cuidado aos idosos no domicílio no contexto da estratégia de saúde da família Rev Gaúch Enferm 2008;29(1):83-9.

8. Ministério da Saúde, Departamento de Informática do Sistema Único de Saúde. Informações de saúde: demográficas e socioeconômicas. [monografia na internet]. Brasília; Ministério da Saúde; 2009. [acessado 10 maio 2009].
Disponível em: < http://tabnet.datasus.gov.br/cgi/tabcgi. exe?ibge/cnv/poprs.def $>$

9. Ministério da Saúde, Secretaria de Vigilância em Saúde, Departamento de Vigilância Epidemiológica. Doenças infecciosas e parasitárias: guia de bolso. 6. ed. Brasília: Ministério da Saúde; 2006.

10. Zornitta M. Os novos idosos com AIDS: sexualidade e desigualdade à luz da bioética. Rio de Janeiro. Dissertação [Mestrado em Saúde Pública]- Escola Nacional de Saúde Pública Sérgio Arouca, Fundação Oswaldo Cruz; 2008.

11. Ministério da Saúde, Secretária de Vigilância em Saúde. Programa Nacional de DST e AIDS. Controle das doenças sexualmente transmissíveis: manual de bolso. 2. ed. Brasília: Ministério da Saúde; 2006.

12. Pinheiro RS, Torres TZG. Análise exploratória de dados. In: Medronho RA, Carvalho DM, Bloch KV, Luiz RR, Werneck GL. Epidemiologia. São Paulo: Atheneu; 2002. p. 227-44.

13. Ministério da Saúde (Brasil). Resolução 196/96 de 10 de outubro de 1996. Diretrizes e Normas Regulamentadoras de Pesquisa em Seres Humanos [resolução na internet]. Diário Oficial da União 10 out 1996 [acessado 08 nov 2008]. Disponível em: <http://www.bioetica.ufrgs.br/ res196/96.htm > 